\title{
Signos radiológicos. Esófago en cascanueces/sacacorchos en el espasmo esofágico
}

\author{
Dres. Horacio Saavedra $M^{(1)}$, Rolando Cocio $A^{(2)}$, Claudio Cortés $A^{(3)}$.
}

1. Becado de Radiología, Centro de Imagenología, Hospital Clínico Universidad de Chile. Santiago - Chile.

2. Becado de Radiología, Centro de Imagenología, Hospital Clínico Universidad de Chile. Santiago - Chile.

3. Radiólogo, Profesor Titular Facultad de Medicina Universidad de Chile. Centro de Imagenología, Hospital Clínico Universidad de Chile. Santiago - Chile.

\section{Nutcracker/corkscrew esophagus in the esophageal spasm}

Abstract: The nutcracker esophagus, also known as corkscrew esophagus, describes the abnormally segmented appearance of the esophagus in cases of esophageal spasm, displayed in the double contrast study. Normally the primary peristalsis extends through the esophagus like a coordinated contraction and relaxation wave. In the esophageal spasm multiple segments contract simultaneously and there is an alteration of the normal relaxation. Symptoms include chest pain, dysphagia, or both.

Keywords: Corkscrew, Esophagus, Fluoroscopy, Motility disorders, Nutcracker, Spasm.

Resumen: El esófago en cascanueces, también conocido como esófago en sacacorchos, describe la apariencia anormalmente segmentada del esófago en los casos de espasmo esofágico, visualizada en el estudio con doble contraste. Normalmente el peristaltismo primario se extiende a través del esófago como una onda coordinada de contracción y relajación. En el espasmo esofágico múltiples segmentos se contraen simultáneamente y hay una alteración de la relajación normal. Los síntomas incluyen dolor en el pecho, disfagia o ambos.

Palabras clave: Cascanueces, Esófago, Espasmo, Fluoroscopía, Sacacorchos, Trastornos de la motilidad.

Saavedra H, Cocio R, Cortés C. Signos radiológicos. Esófago en cascanueces/sacacorchos en el espasmo esofágico. Rev Chil Radiol 2015; 21(1): 22-25.

Contacto: Dr. Horacio Saavedra / dr.hsaavedra@gmail.com

Trabajo recepcionado el 26 de julio de 2014. Aceptado para publicación el 13 de octubre de 2014.

\section{Aspecto imagenológico}

El signo del esófago en cascanueces corresponde a la visualización, en un estudio de doble contraste del esófago, del cambio en la forma normal de la columna de bario, con ondulaciones que representan múltiples segmentos contraídos de forma simultánea y descoordinada en el esófago torácico (ondas terciarias), sin observarse la relajación que implica el peristaltismo normal ${ }^{(1-3)}$.

Tradicionalmente, estas alteraciones han sido correlacionadas con el espasmo esofágico difuso ${ }^{(4,5)}$ (Figura 1).

\section{Significado}

El esófago puede ser causa de dolor torácico. A fines de la década del ' 70 , se describió un subgrupo de estos trastornos motores esofágicos (TME) inespecíficos y se les dio el nombre de "esófago en cascanueces"(6). Se definió así un síndrome consistente en dolor al pecho y/o disfagia en asociación con una presión peristáltica elevada del esófago distal (en ese entonces se definió una amplitud contráctil distal media $>120 \mathrm{mmHg})^{(6)}$.

Los TME tienen como síntomas principales la disfagia y el dolor torácico ${ }^{(3,7)}$. Estos se relacionan con disfunciones del esfínter esofágico inferior y/o alteraciones en la peristalsis del esófago ${ }^{(8)}$. Generalmente se clasifican en TME primarios (fenómeno aislado) y secundarios (en relación a patologías como esclerosis sistémica, diabetes mellitus, enfermedad de chagas y enfermedad por reflujo gastroesofágico) $)^{(9)}$.

Hasta hace poco, los TME primarios que no cumplían con criterios establecidos para otros diagnósticos, se agrupaban como TME no específicos o NEMD (Nonspecific Esophageal Motility Disorder). Los hallazgos radiológicos en NEMD también son inespecíficos e incluyen alteraciones de la peristalsis primaria y contracciones terciarias ${ }^{(10,11)}$.

Actualmente, los TME primarios se dividen en dos grupos: la Acalasia y el resto de Patrones con Motilidad Alterados, siendo definidos por pruebas de motilidad estandarizada (Tabla I(9) $)$. Cabe destacar que estos hallazgos manométricos no son mutuamente excluyentes. 


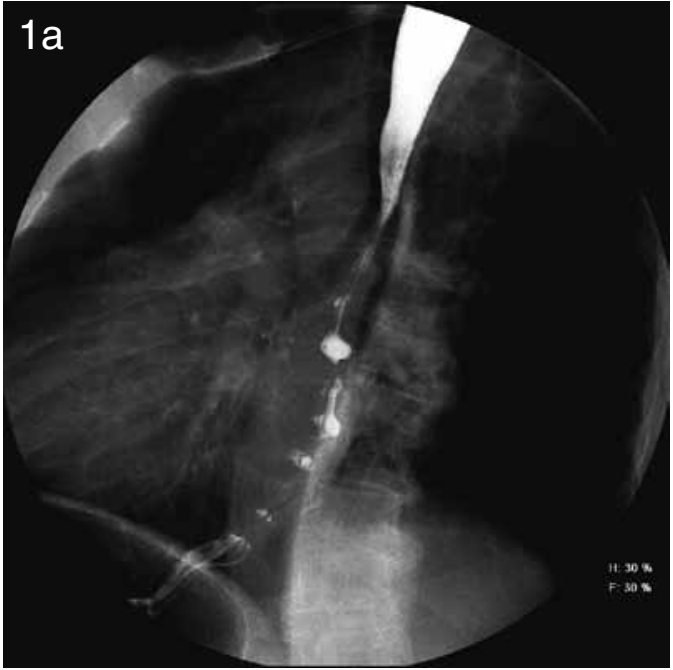

Figura 1 a) Espasmo esofágico difuso. Estudio digestivo baritado con doble contraste que muestra múltiples contracciones no peristálticas de moderada intensidad, con apariencia de esófago en cascanueces. b) Imagen de cascanueces al que hace referencia el signo.

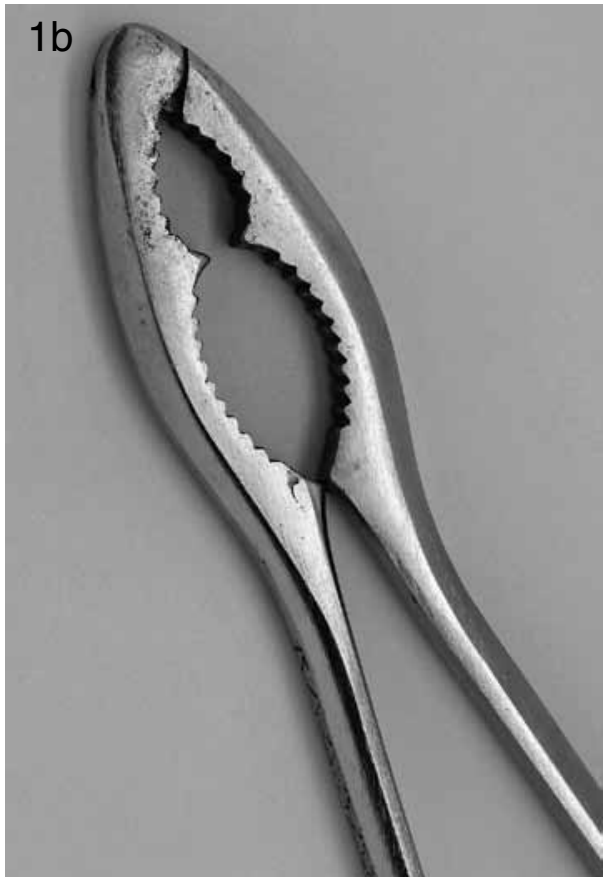

Tabla I. Clasificación de los TME primarios.

\begin{tabular}{|c|c|}
\hline Diagnóstico & Manometría convencional \\
\hline Acalasia (trastorno verdadero) & $\begin{array}{l}\text { Peristalsis distal ausente } \\
\text { Relajación incompleta del EEl (presión residual >8 mmHg) } \\
\text { Presión de reposo del EEl elevada }(>45 \mathrm{mmHg} \text { ) }\end{array}$ \\
\hline $\begin{array}{l}\text { Patrones de motilidad alterados* } \\
\text { Motilidad descoordinada }\end{array}$ & \\
\hline Espasmo esofágico difuso (o distal) & $\begin{array}{l}\text { Contracciones simultáneas ( } \geq 20 \% \text { de los tragos) } \\
\text { Peristalsis intermitente } \\
\text { Contracciones repetitivas ( } \geq 3 \text { peaks) } \\
\text { Contracciones de duración prolongada ( }>6 \mathrm{sec} \text { ) }\end{array}$ \\
\hline \multicolumn{2}{|l|}{ Esófago hipercontráctil } \\
\hline $\begin{array}{l}\text { Peristaltis hipertensiva } \\
\text { ("esófago en cascanueces") } \\
\text { EEl hipertensivo }\end{array}$ & $\begin{array}{l}\text { Mayor amplitud peristáltica distal (promedio>220 mmHg) } \\
\text { Mayor duración peristáltica distal (promedio >6 sec) } \\
\text { Presión de reposo EEI >45 mmHg } \\
\text { Puede haber relajación incompleta del EEl (presión residual }>8 \mathrm{mmHg} \text { ) }\end{array}$ \\
\hline \multicolumn{2}{|l|}{ Esófago hipocontráctil } \\
\hline $\begin{array}{l}\text { Motilidad esofágica inefectiva• } \\
\text { EEl hipotensivo• }\end{array}$ & $\begin{array}{l}\text { Aumento del peristaltismo de baja amplitud ( } \geq 50 \% \text { de los tragos) } \\
\text { Baja amplitud peristáltica distal }(<30 \mathrm{mmHg}) \\
\text { Presión de reposo EEI }<10 \mathrm{mmHg}\end{array}$ \\
\hline Otros & $\begin{array}{l}\text { Contracciones retrógradas } \\
\text { Contracciones de tres puntas } \\
\text { Relajación incompleta aislada del EEl }(>8 \mathrm{mmHg})\end{array}$ \\
\hline $\begin{array}{l}\text { EEI: esfínter esofágico inferior. } \\
\text { * Se definen como } 2 \text { desviaciones estándar sobre los valores } \\
\text { - Puede ser "secundario" a enfermedad por reflujo gastroesof }\end{array}$ & \\
\hline
\end{tabular}


Radiológicamente, la descripción clásica del espasmo esofágico difuso en los estudios baritados incluye la presencia de contracciones no peristálticas, repetitivas y simultáneas que obliteran el lumen y compartimentan o segmentan el esófago, produciendo la imagen distintiva en cascanueces, sacacorchos, en rosario, o en "shish kebab" ${ }^{1,4,5)}$ (del turco shish: ensartada; kebab: plato con trozos de brochetas similar a un anticucho) (Figura 2).
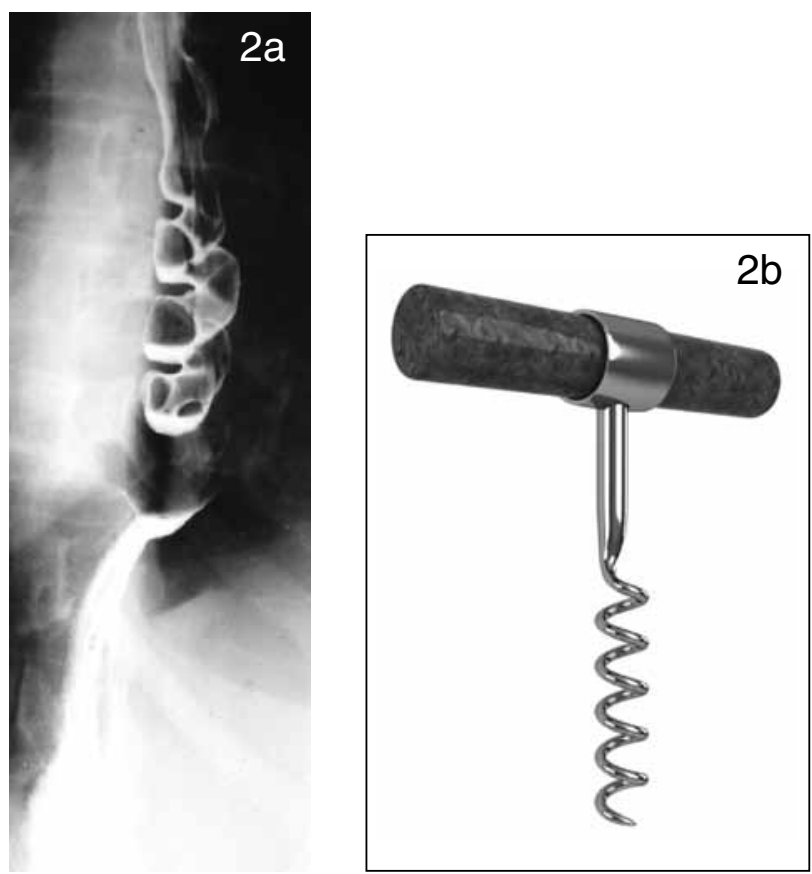

Figura 2. a) Espasmo esofágico difuso. Imagen de estudio baritado doble contraste que muestra múltiples contracciones peristálticas que obliteran el lumen, produciendo la apariencia en sacacorchos. b) Imagen de sacacorchos al que hace referencia el signo.

Sin embargo, Prabhakar A. et $\mathrm{al}^{(2)}$ demostraron que las contracciones no peristálticas que obliteran el lumen y compartimentan el esófago en la fluoroscopía pueden observarse en sólo un $14 \%$ de los pacientes con espasmo esofágico difuso, mientras el $86 \%$ restante presenta espasmos de diferentes magnitudes que no obliteraron el lumen.

Por lo tanto, en los estudios contrastados con bario, el espasmo esofágico difuso se puede definir como la ausencia intermitente o debilitada de peristalsis esofágica primaria, intercalada con contracciones no peristálticas múltiples y simultáneas, de diversa gravedad $^{(1,4,5)}$.

\section{Diagnóstico diferencial}

El principal diagnóstico diferencial es la acalasia vigorosa $^{(12)}$. La acalasia vigorosa se considera una forma menos severa o temprana que la acalasia clásica, con alteraciones motoras similares vistas en la fluoroscopía, además de presentar contracciones simultáneas repetitivas no peristálticas. La dilatación esofágica puede ser mínima. Los pacientes pueden tener disfagia y dolor en el pecho, como también ser asintomáticos, sin embargo, tienen hallazgos radiográficos idénticos (incoordinación motora inespecífica) ${ }^{(12)}$.

\section{Discusión}

La asociación de disfagia y dolor torácico como síntomas en un paciente debe ser objeto de estudio. Tras descartar causas cardíacas, el dolor torácico no cardíaco puede tener un componente en el tubo digestivo, donde las anormalidades estructurales y funcionales en el esófago son causas potenciales.

En el espasmo esofágico difuso, los síntomas pueden variar de leves a severos, pero son clásicamente intermitentes ${ }^{(3)}$. Los episodios de dolor no siempre se correlacionan con las contracciones espásticas, y los pacientes pueden sentir dolor intenso incluso cuando los tragos aparecen fluoroscópicamente normales ${ }^{(3)}$.

El valor del signo del esófago en cascanueces está dado porque permite complementar el diagnóstico manométrico y clínico, corroborando la alteración del peristaltismo normal. El estudio contrastado además permite la detección de anormalidades estructurales del esófago, reconociendo tanto complicaciones que pudieran presentarse del trastorno motor (divertículos, hernias, estenosis distal y acalasia) así como otras anormalidades estructurales del esófago (ulceras, tumores, etc).

Si bien el diagnóstico definitivo está dado por la manometría, el estudio baritado tiene un gran impacto en el diagnóstico global, permitiendo caracterizar de mejor manera los trastornos motores esofágicos.

Los radiólogos debemos tener en cuenta que la gravedad de las contracciones no peristálticas puede variar en pacientes con espasmo esofágico difuso y que la ausencia de un esófago en sacacorchos en los estudios con bario no excluye el diagnóstico.

\section{Bibliografía}

1. Chen YM, Ott DJ, Hewson EG, Richter JE, Wu WC, Gelfand DW, et al. Diffuse esophageal spasm: radiographic and manometric correlation. Radiology 1989; 170: 807-810.

2. Prabhakar A, Levine MS, Rubesin S, Laufer I, Katzka D. Relationship between diffuse esophageal spasm and lower esophageal sphincter dysfunction on barium studies and manometry in 14 patients. AJR Am J Roentgenol 2004; 183(2): 409-413.

3. Adler DG, Romero Y. Primary esophageal motility disorders. Mayo Clin Proc 2001; 76(2): 195-200.

4. Laufer I. Motor disorders of the esophagus. In: Levine MS, ed. Radiology of the esophagus. Philadelphia, PA: Saunders, 1990: 229-246.

5. Ott DJ. Motility disorders of the esophagus. In: Gore RM, Levine MS, eds. Textbook of gastrointestinal radiology, 2nd ed. Philadelphia, PA: Saunders, 2000: 316-318.

6. Benjamin SB, Gerhardt DC, Castell DO. High ampli- 
tude, peristaltic esophageal contractions associated with chest pain and/or dysphagia. Gastroenterology 1979; 77: 478-483.

7. Richter JE. Oesophageal motility disorders. Lancet 2001; 358: 823-828.

8. Martín de Argila C, Boixeda D. Trastornos motores del esófago. Montoro M, García JC. Gastroenterología y Hepatología, Problemas comunes en la práctica clínica. 2da ed. Madrid: Jarpyo Editores SA; 2012. pp. 243-250.

9. Castell, DO. Diffuse esophageal spasm, nutcracker esophagus, and hypertensive lower esophageal sphinc- ter. In: UpToDate, Post TW (Ed), UpToDate, Waltham, MA. (Accessed on May 31, 2014).

10. MS Levine, SE Rubesin, DJ Ott. Update on esophageal radiology. American Journal of Roentgenology 1990; 155: 933-941.

11. Kahrilas PJ. Esophageal motility disorders: current concepts of pathogenesis and treatment. Can J Gastroenterol 2000; 14: 221-231.

12. C. Daniel Johnson, Grant D. Schmit. Mayo Clinic Gastrointestinal Imaging Review. Rochester, MN: Mayo Clinic Scientific Press, 2005. pag 53.

\section{PROTECCIÓN SUPROTECCION ES NUESTRA TAREA ARQuUTECTURA EN IMAGENOLOGí DIAGNOSSTICA.} Toda la protección para su equipo de trabajo (A) 